\title{
An ALTM Digital Height Model Associated with VHR Imagery for an Object-Based Classification of Intra-Urban Targets
}

\author{
Fernando Leonardi ${ }^{1}$, Cláudia Almeida ${ }^{2}$, Leila Fonseca ${ }^{2}$ \\ ${ }^{1}$ Geopixel Ltda, R. Egydio Pinto, 165, São J. Campos, SP \\ Email: fernando@geopx.com.br \\ ${ }^{2}$ Nat. Inst. for Space Research - INPE, São J. Campos, SP \\ E-mail: almeida@dsr.inpe.br, leila@dpi.inpe.br
}

\author{
Lívia Rodrigues Tomás ${ }^{3}$ \\ ${ }^{3}$ Inst. for Research, Administration and Planning - IPPLAN \\ R. Augusto E. Ehlke, 181 \\ São José dos Campos, SP, Brazil \\ Email: liviatomas@gmail.com
}

\begin{abstract}
The study of the urban environment has raised great interest among researchers and practitioners involved with the use of remote sensing, in face of the challenges for its investigation, like the fast and ongoing changes of its structure and the complexity of its targets. New concepts and analyses have been used for mapping the urban space. Object-based analysis and multi-resolution segmentation have been quite efficient in the discrimination of urban targets in high spatial resolution images. In this context, this paper proposes a methodology employing cognitive approaches for the classification of land cover in urban areas using optical orbital and airborne laser scanning data. The results were presented and discussed, indicating a satisfactory accuracy in the generated mapping products, demonstrating the reliability of the methodology for mapping urban land cover.
\end{abstract}

\section{INTRODUCTION}

Urban areas worldwide consist of a huge diversity of land cover materials, and hence, classification methods for the proper discrimination of such targets have been continuously conceived since the beginnings of orbital remote sensing in the early 1970s. This decade was marked by the first generation of remote sensing satellites, namely Landsat 1,2 and 3, which presented a low spatial resolution of approximately $79 \mathrm{~m}$, what severely constrained the exploration of urban studies at that time. Nevertheless, researchers undertook the first detections of urban areas basically through visual interpretation, conducted multitemporal studies for monitoring urban growth and crosschecked this information with the environmental capacity of concerned sites, besides estimating population with very simple regression models taking into account the urban surface.

The second generation started in the mid 1980s and extended until the end of the 1990s, comprising the satellites with medium spatial resolution, like Landsat 5 and SPOT 1, 2, 3 and 4, which spatial resolution increased to $30 \mathrm{~m}$ and $20 \mathrm{~m}$ in the multispectral bands, respectively. This gain in spatial resolution associated with the parallel progress in digital image processing techniques enabled more in-depth studies of urban areas, in which specific zones and targets started being identified, like green areas, residential and industrial zones, main street network, and so on.

In the end of the 1990s and beginning of the 2000s, there was a profusion of the so-called high ( $>4 \mathrm{~m}-10 \mathrm{~m})$ and very high spatial resolution (VHR) sensors ( $0.5 \mathrm{~m} \mathrm{-} 4 \mathrm{~m})$ onboard of satellites like IKONOS-2, QuickBird, World-View 1 and 2, OrbView, Kompsat, GeoEye and others. These sensors not only had their spatial resolution increased but their radiometric resolution as well, what implied in the generation of imagery of massive size. New challenges were then imposed to the digital images processing field, which experienced so-tosay a shift in paradigm at this point, since the platforms developed hitherto did not dispose of enough processing capacity to deal with this huge amount of data, neither the available methods were robust enough to extract information from such complex images endowed with both high radiometric and spatial resolutions.

\section{THE OBJECT-BASED APPROACH FOR CLASSIFICATION}

The object-oriented image analysis, further renamed as object-based image analysis (OBIA), arose as a response to the observed hindrances of conventional digital images processing methods. As explicited in its original terminology, it inherited the basic premises of the object-oriented $(\mathrm{O}-\mathrm{O})$ paradigm from Computer Sciences, where an object, formally defined as an abstract entity (which may or may not have a linkage to the real word phenomena), is regarded as the instance of a class. The class, also an abstract concept, represents a set of objects with common characteristics, known as attributes [1]. Another important O-O concept is inheritance (or generalization), which consists in a mechanism through which a given class in an inferior hierarchical level (subclass) can inherit from another class in a superior level (superclass) its attributes and associated behavior (mathematical function that describes the attribute)[1]. For instance, the subclasses 'trees' and 'grass' inherit from the superclass 'vegetation' its high NDVI values.

In OBIA, the knowledge model adopted for interpreting an image is in principle a semantic network, consisting of a hierarchical structure of classes, to which image objects are associated. Semantic networks are graphs that formalize and represent the knowledge of the human interpreter by means of a set of nodes connected by arcs. The nodes represent concepts, and the arcs the relations among them [2]. The pixel could not actuate as an object, for it does not allow the extraction of attributes. Hence, the region derived from image segmentation plays the role of an object in OBIA and constitutes the 
primitive unit for image classification. OBIA has also enabled parallel advances in image interpretation, like (i) the use of not only statistical attributes, but also geometrical, topological, contextual as well as attributes related to the semantic relationship between classes [3-5]; (ii) the possibility of operating with more than one level of segmentation, that may or may not be associated with a classification [6]; and (iii) refined segmentation algorithms, which incorporated parameter associated to the shape of objects [6].

Although the association between OBIA and high spatial resolution sensors allowed the classification of urban land cover at a detailed level, some confusion between classes still remain for objects with similar spectral response. For this reason, the information on the height of objects is crucial for solving such conflicts, which is rendered possible through the use of digital elevation models of elevated vertical accuracy.

\section{STUDY AREA}

This work is committed to conduct an object-based classification of intra-urban land cover targets using IKONOS2 images and a digital height model obtained with the ALTM 2025 laser scanning sensor for a central sector of Uberlandia city (Fig. 1), located in the southeastern state of Minas Gerais, Brazil.

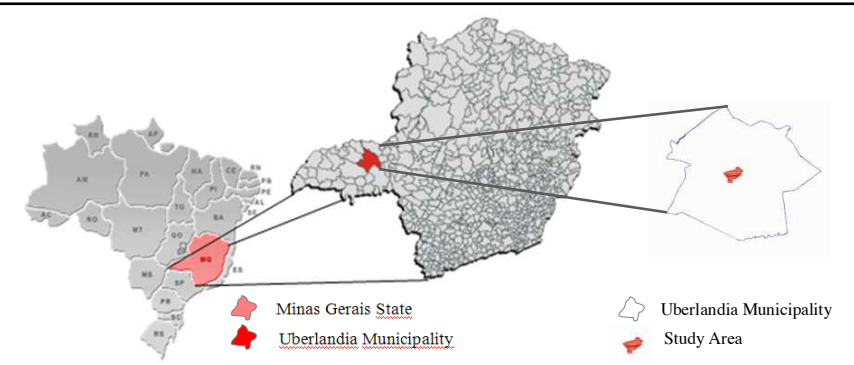

(a)

(b)

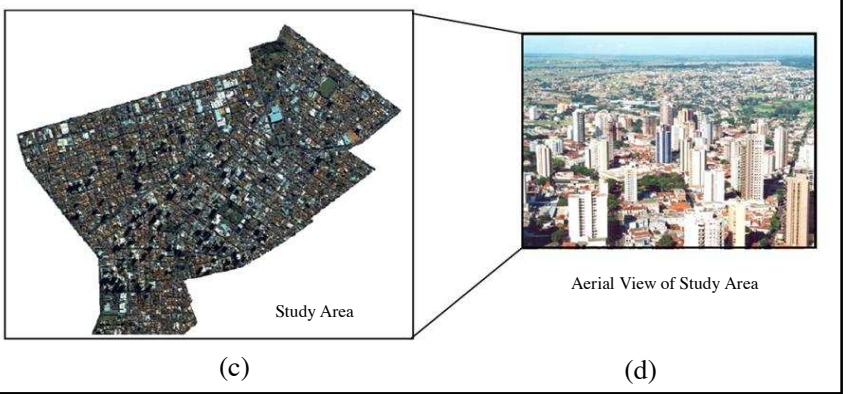

Figure 1. Location of study area. a. Minas Gerais State and Uberlandia Municipality (in red). b. Location of study area inside the municipality. c. Study area (central sector of Uberlandia city) in true color composition of IKONOS-2 images (1B_2G_3R). d. Aerial view of study area.

\section{METHODS}

\section{A. Data Pre-Processing}

Initially, the IKONOS-2 multispectral bands were pansharpened with the respective panchromatic band using the HISmethod with the cubic convolution interpolator. After this procedure, the image was subject to an orthorectifying process, based on GPS points collected in the field and evenly distributed over the study area. In total, 55 GPS points were collected, with an approximate planimetric (horizontal) accuracy of $0.030 \mathrm{~m}$, and an elevation (vertical) accuracy of nearly $0,021 \mathrm{~m}$. All of the points were processed using the UTM projection, South Zone 22, Datum WGS 84, based on the MGUB and UBER stations from the Brazilian Network for Ongoing Monitoring. After the acquisition and processing of field data, the image was orthorectified in absolute mode, using such GPS points, the sensor attitude and ephemeris data (rendered available in the image metadata files), and the elevation data derived from the laser scanning air survey accomplished with ALTM 2025 [7].

The acquired LiDAR data were pre-processed and delivered in ASCII format, containing the information of $\mathrm{X}, \mathrm{Y}$, and $\mathrm{Z}$ coordinates as well as the intensity of the DSM and DTM. A filtering process was applied to the DSM, so as to obtain the DTM using the software TerraScan, available at the Terra Solid platform. TerraScan collects the irregularly spaced $3 \mathrm{D}$ points cloud and by means of its terrain classifier, known as Axelsson's progressive TIN densification algorithm [8-9]. It extracts points directly located on the terrain surface by constructing an iterative triangular irregular network (TIN). It is possible to correct errors where the automatic classification (filtering process) did not present good results through the command "add point to the terrain". Upon basis of this classification result, two files in the TXT format were generated: one containing the DSM and another one the DTM [7]. The subtraction between the DSM and DTM generated the digital height model (DHM), which was actually used in the classification process (Fig. 2).

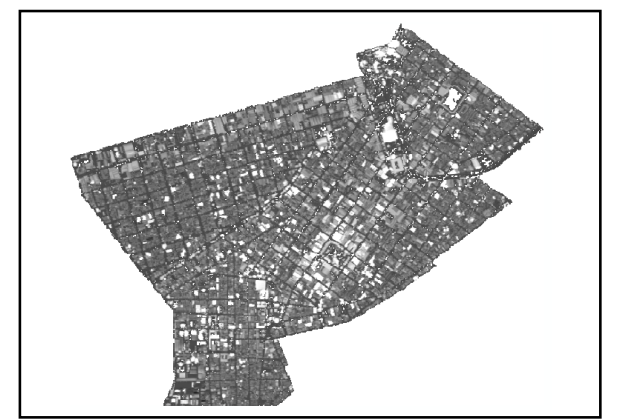

Figure 2. Digital height model (DHM) of the study area.

\section{B. Definition of Segmentation Parameters}

A genetic algorithm implemented in plug-in named SPT, developed by PUC-Rio, was used to identify optimal values, within a given search space, for all segmentation parameters required by the OBIA platform Definiens, i.e. scale factor, weights for each input band, color and shape parameters as well as compactness and smoothness parameters. The most satisfactory value is determined by an objective-function which assesses the degree of agreement between the segmentation results and the reference samples, consisting of segments manually delimited by the interpreter. In mathematical terms, given a set of reference segments $S$, a disparity function $F$, and a vector of parameters $P$, the genetic algorithm (GA) aims at finding the optimal set of segmentation parameters $P_{\text {opt }}$ defined as in (1) [11]: 


$$
P_{\text {opt }}=\arg _{P} \min ([F(S, P)]) .
$$

The user has to define the GA internal parameters, like number of experiments, population size, number of generations, initial and final gap. Nevertheless, SPT has as its default parameters values that have proved to generate the best results. Data are expected to converge after a certain amount of generations, producing a segmentation at the end.

\section{The Semantic Network Design}

The parameters provided by SPT were adopted for segmenting the four pan-sharpened IKONOS II image bands in the Definiens Developer 7.0.4. In order to build the hierarchical semantic network, the data mining algorithm C4.5 [11] was used. This algorithm builds decision trees based on training

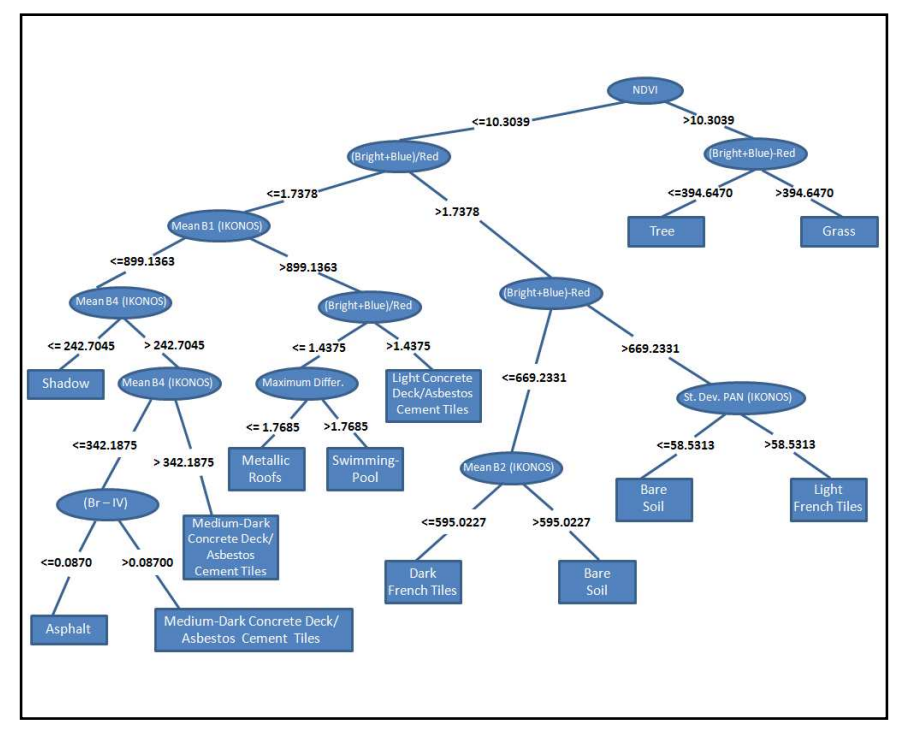

Figure 3.Decision tree using IKONOS-2 imagery only.

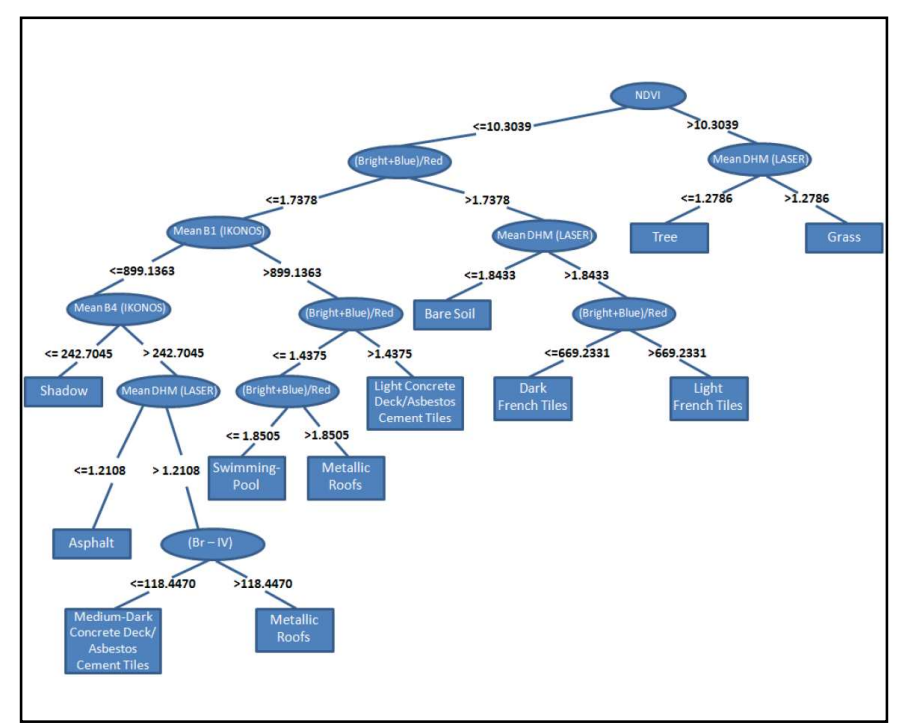

Figure 5. Decision tree using IKONOS-2 imagery and the LiDAR DHM. samples. It is aimed at building compact and simple trees, for they are more easily understood and present a better performance [11]. In this work, eleven classes were defined: bare soil, light French tiles, dark French tiles, metallic roofs, swimming pool, light concrete deck or light asbestos cement tiles, medium to dark concrete deck or medium to dark asbestos cement tiles, asphalt, shadow, trees, and grass. All attributes existent in the Definiens platform together with customized ones related to arithmetic transforms of image bands were added to the training set, what totalized 355 attributes, exported as file with CSV extension. The input dataset was subject to a preliminary filtering for removing noise and inconsistencies. The number of training samples per class was very diverse, but they tried to be representative of the spectral and textural heterogeneity of the concerned classes. A minimum of 55 objects (sample units) was set to be considered during the decision tree processing. Two OBIA experiments

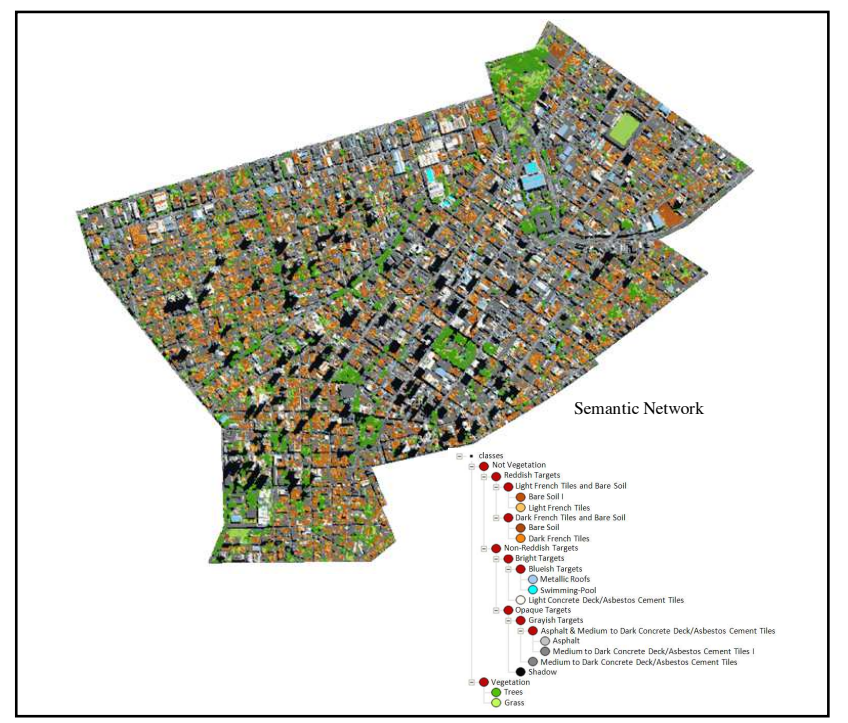

Figure 4. Classification using IKONOS-2 imagery only.

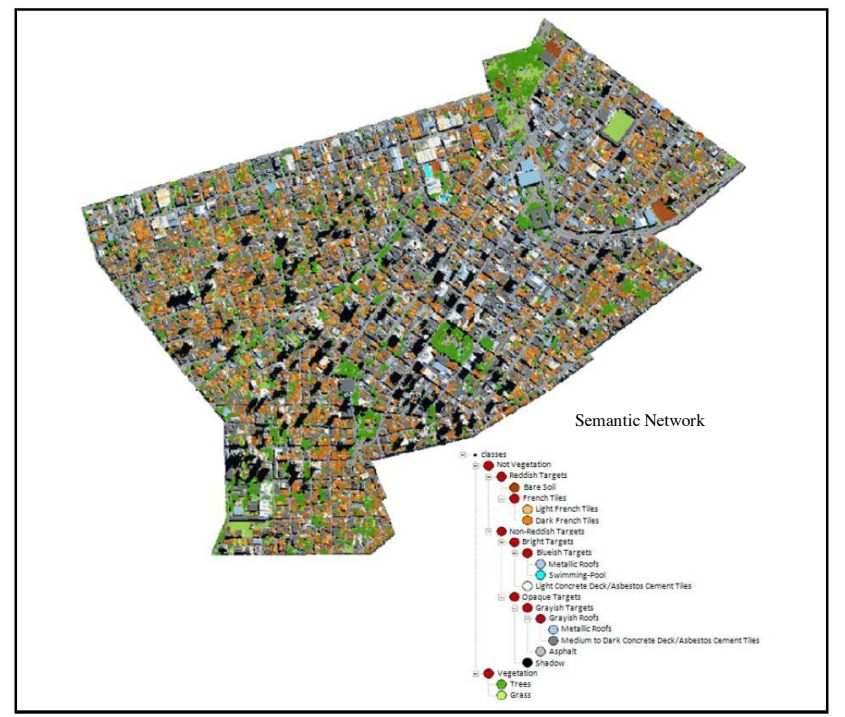

Figure 6. Classification using IKONOS-2 imagery and the LiDAR DHM. 
were conducted: including and excluding the LiDAR-derived DHM, in order to evaluate the impact of the elevation information in the classification accuracy.

\section{RESULTS AND DISCUSSION}

After a certain number of consecutive training experiments, the final decision tree was produced for each experiment, relying on samples generated with the optimal segmentation parameters provided by SPT, namely a scale factor of 11 , a color factor of 0.57 , a compactness factor of 0.64 , and a weight of 0.06 for the blue band, of 0.52 for the green band, and of 0.42 for the red band. In the first experiment, without the DHM, nine attributes out of the 355 initially selected were used (Fig. 3), while in the second one, with the DHM, only five attributes were effectively employed (Fig. 5). These decision trees were then converted into hierarchical semantic networks inside the Definiens platform and generated the respective urban land cover classifications (Fig. 4 and 6).

In both cases, the decision trees showed a concise and logical structure, but the tree that did not employ the DHM as an input band used nearly twice as many attributes in comparison to the tree that included the DHM. The first tree, which disregarded the elevation information, also showed two cases of duplicated end-nodes (bare soil and medium to dark concrete deck or medium to dark asbestos cement tiles), while in the latter one, this happened only once (metallic roofs). The most important aspect to be highlighted, however, is the fact

TABLE I. ERROR MATRIX AND AGREEMENT INDICES FOR THE OBJECT-BASED CLASSIFICATION USING IKONOS-2 IMAGERY ONLY.

\begin{tabular}{|c|c|c|c|c|c|c|c|c|c|c|c|c|c|}
\hline \multirow{2}{*}{\multicolumn{2}{|c|}{ CLASSES }} & \multicolumn{11}{|c|}{ References } & \multirow[b]{2}{*}{ Total } \\
\hline & & $\mathrm{A}$ & B & $\mathrm{C}$ & $\mathrm{D}$ & $\mathrm{E}$ & $\mathrm{F}$ & $\mathrm{G}$ & $\mathrm{H}$ & I & $\mathrm{J}$ & $\mathrm{K}$ & \\
\hline \multirow{12}{*}{ 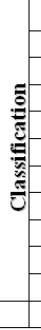 } & A & 1 & 0 & 0 & 0 & 0 & 0 & 0 & 0 & 0 & 0 & 0 & 1 \\
\hline & B & 0 & 5 & 0 & 0 & 11 & 8 & 0 & 1 & 1 & 1 & 0 & 27 \\
\hline & $\mathrm{C}$ & 0 & 0 & 35 & 3 & 0 & 0 & 0 & 1 & 0 & 1 & 7 & 47 \\
\hline & $\mathrm{D}$ & 0 & 0 & 10 & 6 & 0 & 0 & 0 & 0 & 0 & 0 & 0 & 16 \\
\hline & $E$ & 0 & 4 & 0 & 0 & 13 & 1 & 0 & 0 & 0 & 0 & 0 & 18 \\
\hline & $\mathrm{F}$ & 0 & 3 & 0 & 0 & 0 & 30 & 0 & 2 & 0 & 0 & 17 & 52 \\
\hline & $\mathrm{G}$ & 0 & 0 & 0 & 0 & 0 & 0 & 5 & 2 & 5 & 3 & 0 & 15 \\
\hline & $\mathrm{H}$ & 0 & 0 & 0 & 0 & 0 & 0 & 1 & 23 & 0 & 11 & 2 & 37 \\
\hline & I & 0 & 0 & 0 & 0 & 0 & 0 & 2 & 7 & 24 & 8 & 0 & 41 \\
\hline & $\mathrm{J}$ & 0 & 0 & 0 & 0 & 0 & 3 & 5 & 42 & 9 & 88 & 25 & 172 \\
\hline & $\mathrm{K}$ & 0 & 0 & 0 & 0 & 0 & 0 & 0 & 7 & 0 & 0 & 67 & 74 \\
\hline & Total & 1 & 12 & 45 & 9 & 24 & 42 & 14 & 85 & 39 & 112 & 118 & 500 \\
\hline & Glob & $\mathbf{A C C}$ & Iracy & 0.592 & & & pa In & $\mathrm{ex}: 0$ & 376 & & iance o & appa: & 00062 \\
\hline
\end{tabular}

* A = swimming pool; $\mathrm{B}=$ bare soil; $\mathrm{C}=$ trees; $\mathrm{D}=$ grass; $\mathrm{E}=$ light French tiles; $\mathrm{F}=$ dark French tiles; $\mathrm{G}=$ A $=$ swimming pool $; \mathrm{B}=$ bare soil; $\mathrm{C}=$ trees; $\mathrm{D}=$ grass; $\mathrm{E}=$ light French tiles; $\mathrm{F}=$ dark French tiles; $\mathrm{G}=$
metallic roofs; $\mathrm{H}=$ asphalt; $\mathrm{I}=$ light concrete deck or light asbestos cement tiles; $\mathrm{I}=$ medium to dark concrete deck or medium to dark asbestos cement tiles; $\mathrm{K}=$ shadow.

TABLE II. ERROR MATRIX AND AGREEMENT INDICES FOR THE OBJECT-BASED CLASSIFICATION USING IKONOS-2 AND THE LIDAR DHM.

\begin{tabular}{|c|c|c|c|c|c|c|c|c|c|c|c|c|c|}
\hline \multirow{2}{*}{\multicolumn{2}{|c|}{ CLASSES }} & \multicolumn{11}{|c|}{ References } & \multirow{2}{*}{ Total } \\
\hline & & A & B & $\mathrm{C}$ & $\mathrm{D}$ & $\mathrm{E}$ & $\mathrm{F}$ & $\mathrm{G}$ & $\mathrm{H}$ & I & $\mathrm{J}$ & $\mathrm{K}$ & \\
\hline \multirow{12}{*}{ 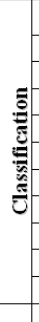 } & A & 1 & 0 & 0 & 0 & 0 & 0 & 0 & 0 & 0 & 0 & 0 & 1 \\
\hline & B & 0 & 8 & 0 & 0 & 0 & 3 & 0 & 2 & 1 & 0 & 4 & 18 \\
\hline & $\mathrm{C}$ & 0 & 0 & 39 & 7 & 0 & 0 & 0 & 0 & 0 & 1 & 0 & 47 \\
\hline & $\mathrm{D}$ & 0 & 0 & 4 & 12 & 0 & 0 & 0 & 0 & 0 & 0 & 0 & 16 \\
\hline & E & 0 & 0 & 0 & 0 & 11 & 4 & 0 & 0 & 6 & 0 & 0 & 21 \\
\hline & $\mathrm{F}$ & 0 & 0 & 0 & 0 & 0 & 40 & 0 & 1 & 3 & 3 & 10 & 57 \\
\hline & $\mathrm{G}$ & 0 & 0 & 0 & 0 & 0 & 0 & 18 & 2 & 2 & 4 & 4 & 30 \\
\hline & $\mathrm{H}$ & 0 & 1 & 0 & 0 & 0 & 0 & 1 & 62 & 5 & 3 & 8 & 80 \\
\hline & I & 0 & 0 & 0 & 0 & 0 & 0 & 0 & 1 & 30 & 7 & 0 & 38 \\
\hline & $\mathrm{J}$ & 0 & 0 & 2 & 0 & 0 & 5 & 0 & 6 & 4 & 94 & 6 & 117 \\
\hline & $\mathrm{K}$ & 0 & 0 & 0 & 0 & 0 & 0 & 1 & 7 & 0 & 0 & 67 & 75 \\
\hline & Total & 1 & 9 & 45 & 19 & 11 & 52 & 20 & 81 & 51 & 112 & 99 & 500 \\
\hline \multicolumn{14}{|c|}{ Global Accuracy: 0.7640} \\
\hline
\end{tabular}

that the second tree produced a classification with a considerably higher accuracy in relation to the first one, demonstrating the remarkable discriminative power of the DHM. As shown in Table I, the global accuracy was only $59.2 \%$ and the Kappa index was approximately 0.54 in the first experiment, whereas the global accuracy rose to $76.4 \%$ and the Kappa index to $73 \%$ in the second experiment (Table II).

\section{CONCLUSIONS}

It is worth mentioning that the mean of the DHM has been used to differentiate trees from grass, bare soil from dark and light French tiles, and medium to dark concrete deck or asbestos cement tiles from asphalt, as expected. Adding height information derived from laser scanning to the multispectral images helped in the discrimination between targets with similar spectral behavior but diverging elevation values. In operational terms, the elaboration of the semantic network by means of data mining proved to be advantageous, for allowing the automation of both the attributes selection and the decision rules definition, demonstrating hence to be less vulnerable to the interpreter's subjectivity.

\section{ACKNOWLEDGMENT}

The authors would like to express their gratitude to the Coordination for the Capacity Building of Graduate Personnel - CAPES, for sponsoring this research.

\section{REFERENCES}

[1] P. Dall'Oglio, "Programming with Object-Orientation". São Paulo: Novatec, 2009. 2. ed. 574 p.

[2] R. Tönjes, S. Growe, J. Bückner, C.-E. Liedtcke, "Knownledge-based interpretation of remote sensing images using semantic nets", Photogrammetric Engineering \& Remote Sensing, Vol. 65, No. 7, 1999, pp. 811-821.

[3] K. Navulur, "Multispectral image analysis using the object-oriented paradigm”. Boca Raton: Taylor \& Francis Group, 2006. 165 p.

[4] P. R. Marpu, "Geographic Object-based Image Analysis", PhD Thesis, Faculty of Geosciences, Geo-Engineering and Mining Technische Universität Bergakademie Freiberg, Germany, 2009.

[5] F. F. Camargo, T. G. Florenzano, C. M. Almeida, C. G. Oliveira, R. Q. Feitosa, "Object-based analysis and ASTER/Terra data for classifying relief units", Boletim de Ciências Geodésicas, Vol. 15, No. 1, 2009. pp. 81-102.

[6] C. N. Francisco, C. M. Almeida, "Evaluating the performance of statistical and textural attributes for an object-based land cover classification", Boletim de Ciências Geodésicas, Vol. 18, No. 2, 2012 pp. 302-326.

[7] F. Leonardi, C. Almeida, L. Fonseca, L. Tomás, P. G. Albuquerque, C. Oliveira, "Elevation Accuracy Assessment of a DSM and DTM Generated for an Urban Area from the ALTM 2025 Airborne Laser Scanning Sensor", In: Proceedings of 2012 Second International Workshop on Earth Observation and Remote Sensing Applications EORSA, 2012.

[8] P. Axelsson, "DEM generation from laser scanning data using adaptive TIN models," ISPRS International Archives of Photogrammetry and Remote Sensing, vol. 33, part B4/1, pp. 110-117, 2000.

[9] TerraSolid, "TerraScan user's guide," 2010, Available at http://www.terrasolid.fi.

[10] C. M. B. Fredrich, R. Q. Feitosa, "Automatic adaptation of segmentation parameters applied to inhomogeneous objects detection", In: Proceedings of GEOBIA, 2008.

[11] R. Quinlan, "C4.5: programs for machine learning”. San Francisco: Morgan Kaufmann, 1993. 